\title{
Integrable Systems and Quantum Groups
}

\author{
Itzhak Roditi \\ Centro Brasileiro de Pesquisas Físicas \\ Rua Dr. Xavier Sigaud 150, Rio de Janeiro, 22290-180, Brazil
}

Received 7 January, 2000

We present some aspects of the study of quantum integrable systems and its relation to quantum groups.

\section{Introduction}

The main purpose of this lecture is to present the study of quantum integrable systems and its interplay with the study of quantum groups. The history of quantum integrable systems begins, following a certain historical path, with the first attempts of W. Heisenberg to develop a microscopic theory that could explain ferromagnetism [1]. Later on a method was proposed by H. Bethe [2] in order to obtain the spectrum of the isotropic Heisenberg model. This approach is called the Bethe ansatz and it gives the parameterization of the eigenvectors through a set of equations, called the Bethe ansatz equations establishing the solvability of the model. Since then a steady evolution has taken place, going through the works of L. Onsager [3], C.N. Yang and C. P. Yang [4], R. Baxter [5] and many others, culminating with the advent of the Quantum Inverse Scattering Method (QISM)[6]. The time interval between Bethe's solution and the QISM is of almost fifty years. The QISM was developed to investigate integrable systems in quantum field theory and quantum statistical physics and it was largely based on an algebraic viewpoint. It provided a unified framework for treating the previous approaches used to solve integrable systems, in theoretical and mathematical physics. Moreover, it is due to the QISM that quantum groups made their appearance in the physics scenario.

The study of quantum groups, or quantum algebras $[7,8]$ is crucial to the understanding of integrable systems as well as the development of new integrable models. It also grew as an independent area in mathematical physics showing interesting connections with other mathematical subjects such as knot theory and non-commutative geometry.

At this point due to space and time limits, inherent to a lecture, it would be useful to make reference to a certain number of excellent reviews where one can find a deeper insight into the QISM and quantum groups, as well as a comprehensive list of references [9].

In the next section the QISM is introduced and quantum groups are defined. The choice of presentation follows an algebraic approach, giving a quite general and model independent framework.

In section III we show how to obtain multiparamet- ric quantum spin chains still based on algebraic results. The motivation of that section is related to the study of the effect of boundary conditions in models describing systems of correlated electrons. In particular we establish a correspondence between twisted boundary conditions and multiparametric models [10, 11, 12]

Section IV is devoted to mentioning some other developments and to draw some indications about future work.

Before we proceed some acknowledgments are in order. The present work has much profited from the discussions with my colleagues from CBPF, Marco RMonteiro and Ligia Rodrigues as well as with my collaborators and friends, Angela Foerster, Katrina Hibberd, Antonio Lima-Santos and Jon Links, whose lecture notes [13] have been extremely useful.

\section{Quantum Inverse Scattering Method: a telegraphic ver- sion}

Let us introduce here in a very brief way some of the algebraic structure defining the QISM. Let $\mathcal{T}_{j}^{i}(\lambda), 1 \leq$ $i, j \leq n$, be $n^{2}$ generators of an abstract algebra $\mathcal{A}$ and $\bar{\lambda} \in \mathbf{C}$ is called the spectral parameter. Consider an invertible operator

$$
R(\lambda) \in \operatorname{End}(V \otimes V),
$$

where $V$ is an n-dimensional vector space. $\mathrm{R}$ may be written in component form as

$$
R(\lambda)=\sum_{i j k l} R_{i k}^{j l}(\lambda) e_{j}^{i} \otimes e_{l}^{k} .
$$

One can set

$$
\mathcal{T}(\lambda)=\sum_{i j} e_{j}^{i} \otimes \mathcal{T}_{i}^{j}(\lambda)
$$

and impose generalized commutation relations in $\mathcal{A}$ via

$$
\begin{aligned}
& R_{12}(\lambda-\mu) \mathcal{T}_{13}(\lambda) \mathcal{T}_{23}(\mu) \\
= & \mathcal{T}_{23}(\mu) \mathcal{T}_{13}(\lambda) R_{12}(\lambda-\mu),
\end{aligned}
$$


where the subscripts refer to the embeddings in End $(V \otimes V) \otimes A . R_{i j}^{k l}(\lambda)$ may be seen to give the structure constants of the algebra $\mathcal{A}$, if one writes (1) as

$$
R_{i k}^{j l}(\lambda-\mu) \mathcal{T}_{j}^{p}(\lambda) \mathcal{T}_{l}^{q}(\mu)=\mathcal{T}_{k}^{j}(\mu) \mathcal{T}_{i}^{l}(\lambda) R_{l j}^{p q}(\lambda-\mu) .
$$

From (1) and the cyclic property of the trace one can show that the elements

$$
\begin{gathered}
\mathcal{T}_{13}(\lambda) \mathcal{T}_{23}(\mu)=R_{12}^{-1}(\lambda-\mu) \mathcal{T}_{23}(\mu) \mathcal{T}_{13}(\lambda) R_{12}(\lambda-\mu) \\
\tau(\lambda)=(\operatorname{tr} \otimes I) \mathcal{T}(\lambda)=\sum_{i=1}^{n} \mathcal{T}_{i}^{i}(\lambda)
\end{gathered}
$$

of the algebra $\mathcal{A}$ commute

$$
[\tau(\lambda), \tau(\mu)]=0, \forall \lambda, \mu \in \mathbf{C} .
$$

The fact that $\tau(\lambda)$ provides a one-parameter family of mutually commuting elements of the algebra $\mathcal{A}$ is deeply related to the definition of integrability as we shall discuss later. A sufficient condition for $\mathcal{A}$ to be associative and then be able to obtain matrix representations is that

$$
\begin{aligned}
& R_{12}(\lambda-\mu) R_{13}(\lambda-w) R_{23}(v-w) \\
= & R_{23}(v-w) R_{13}(\lambda-w) R_{12}(\lambda-\mu),
\end{aligned}
$$

on the space End $(V \otimes V \otimes V)$, which is the celebrated Yang-Baxter equation. We call the algebra $\mathcal{A}$ defined in such a way a Yang-Baxter algebra or a Yangian algebra. The fact that $R(\lambda)$ must satisfy the Yang-Baxter equation provides us with the adjoint representation,

$$
\pi\left(\mathcal{T}_{i}^{j}(\lambda)\right)=\sum_{k, l} R_{i k}^{j l}(\lambda) e_{l}^{k} .
$$

Furthermore, the Yangian algebra $\mathcal{A}$ has a structure of a bi-algebra which can be seen from the existence of the following algebra homomorphisms, the co-product

$$
\Delta: A \rightarrow A \otimes A
$$

defined by

$$
\Delta\left(\mathcal{T}_{i}^{j}(\lambda)\right)=\sum_{k} \mathcal{T}_{k}^{j}(\lambda) \otimes \mathcal{T}_{i}^{k}(\lambda)
$$

and the co-unit

$$
\begin{gathered}
\epsilon: A \rightarrow \mathbf{C} \\
\epsilon\left(\mathcal{T}_{i}^{j}(\lambda)\right)=\delta_{i}^{j} .
\end{gathered}
$$

The significance of the co-product $\Delta$ is that it allows us to build tensor product representations of $\mathcal{A}$.

If $\pi_{1}, \pi_{2}$ are two representations of $\mathcal{A}$ which act on the spaces $V_{1}, V_{2}$ respectively then

$$
\left(\pi_{1} \otimes \pi_{2}\right)\left(\mathcal{T}_{i}^{j}(\lambda)\right)=\pi_{1}\left(\mathcal{T}_{i}^{k}(\lambda)\right) \otimes \pi_{2}\left(\mathcal{T}_{k}^{j}(\lambda)\right)
$$

gives a representation on $V_{1} \otimes V_{2}$. We may also use the opposite co-product $\bar{\Delta}$ given by

$$
\bar{\Delta}\left(\mathcal{T}_{i}^{j}(\lambda)\right)=\mathcal{T}_{i}^{k}(\lambda) \otimes \mathcal{T}_{k}^{j}(\lambda)
$$

which is also an algebra homomorphism.

\section{II.1 Quasi-triangular Hopf algebras}

In the above we have defined the Yangian algebra $\mathcal{A}$ and seen that it has a bi-algebra structure. The discussed properties are part of the construction of quantum groups, or quasi-triangular Hopf algebras. A Hopf algebra can be defined as a bi-algebra that possesses an antihomomorphism

$$
S: \mathcal{A} \rightarrow \mathcal{A},
$$

such that

$$
m(S \otimes I) \Delta(a)=m(I \otimes S) \Delta(a)=i \cdot \epsilon(a),
$$

where $m$ and $i$ are respectively the multiplication and unit element maps on $\mathcal{A}$, defined by

$$
m(a \otimes b)=a \cdot b
$$

and

$$
i(\alpha)=\alpha I,
$$

$\forall a \in \mathcal{A}$ and $\alpha \in \mathbf{C}$.

The key ingredient in the definition of a quantum group is the existence of an element that establishes a relation between the co-product and the opposite coproduct developing an isomorphism between the tensor product $\pi_{1} \otimes \pi_{2} \rightarrow \pi_{2} \otimes \pi_{1}$ of two representations. More precisely, a Hopf algebra $\mathcal{A}$ is quasi-triangular if there exists an invertible element $\mathcal{R} \in \mathcal{A} \otimes \mathcal{A}$, called the universal $\mathrm{R}$-matrix, that satisfies the following relations,

$$
\begin{aligned}
\bar{\Delta}(a) \mathcal{R} & =\mathcal{R} \Delta(a), \forall a \in \mathcal{A} \\
(I \otimes \Delta) \mathcal{R} & =\mathcal{R}_{13} \mathcal{R}_{12} \\
(\Delta \otimes I) \mathcal{R} & =\mathcal{R}_{13} \mathcal{R}_{23}
\end{aligned}
$$

and

$$
(S \otimes I) \mathcal{R}=\left(I \otimes S^{-1}\right) \mathcal{R}=\mathcal{R}^{-1} .
$$

As a result of the above relations the universal R-matrix is a solution of the Yang-Baxter equation,

$$
\mathcal{R}_{12} \mathcal{R}_{13} \mathcal{R}_{23}=\mathcal{R}_{23} \mathcal{R}_{13} \mathcal{R}_{12}
$$

One of best known examples of a quantum group is $U_{q}\left(s l_{2}\right)$, which is associated to the anisotropic Heisenberg model. Much of the above discussion can be extended to superalgebras and to affine (super)algebras. The universal R-matrix $\mathcal{R}$ presented above does not depend on a spectral parameter. A Yang-Baxter equation that depends on a spectral parameter may be obtained for quantum affine (super)algebras and its solution provides a spectral parameter dependent R-matrix, by use of a loop representation, this procedure called Baxterization [14] will not be discussed here. We just notice 
that through Baxterization it is possible to obtain $\mathrm{R}$ matrices related to new integrable systems.

\section{II.2 Integrable systems: an overview}

Using the representations of a Yangian algebra $\mathcal{A}$ one is in position to construct a one-dimensional abstract integrable quantum system. For this purpose let us define the Lax operator as the adjoint representation

$$
L_{i}^{j}(\lambda)=\sum_{k, l} R_{i k}^{j l}(\lambda) e_{l}^{k} .
$$

Where the R-matrix satisfies the Yang-Baxter equation on $V \otimes V$ for a given vector space $V$. Those Laxoperators are local matrix operators, meaning that they act on some internal space at a particular site. On the $(N+1)$-fold tensor product space we may construct the monodromy matrix

$$
T(\lambda)=L_{0 N}(\lambda) L_{0(N-1)}(\lambda) \ldots . L_{01}(\lambda),
$$

which gives a representation of $\mathcal{A}$ on the space $V^{\otimes N}$ as

$$
R_{12}(\lambda-\mu) L_{13}(\lambda) L_{23}(\mu)=L_{23}(\mu) L_{13}(\lambda) R_{12}(\lambda-\mu),
$$

where the subscript 3 refers to the space $V^{\otimes N}$ and the space to which the subscript 0 refers is usually called the auxiliary space. One also defines, in this representation, the transfer matrix

$$
t(\lambda)=\operatorname{tr}_{0} T(\lambda)=\pi(\tau(\lambda))=\pi\left(\sum_{i} \mathcal{T}_{i}^{i}(\lambda)\right)
$$

and it is easy to verify that the transfer matrices commute.

$$
[t(\lambda), t(\mu)]=0, \forall \lambda, \mu \in \mathbf{C} .
$$

Let us now expand the transfer matrix in a power series

$$
t(\lambda)=\sum_{k} c_{k} \lambda^{k}
$$

from the commutation relations it follows that

$$
\left[c_{k}, c_{j}\right]=0 \quad \forall k, j
$$

and one may interpret the operators $c_{k}$ as conservation laws for some abstract one-dimensional quantum system acting on the space $V^{\otimes N}$. We consider that a model is integrable when the number of conserved quantities and the number of degrees of freedom of the system are equal.

From the intertwining relation for the monodromy matrix when $\lambda=\mu$ it is reasonable to expect that $R(0)$ can be made proportional to the permutation operator $P$, with that in mind it may be possible to extract from the transfer matrix some operators for which one can give a physical interpretation.

In particular, assuming that the R-matrix can be normalized so that $R(0)=P$, one has

$$
t(0)=\operatorname{tr}_{0} L(0)=P_{1 N} P_{1(N-1)} \ldots P_{12} .
$$

This operator will act as a shift operator on a periodic lattice of $N$ sites.

Also usually one defines the Hamiltonian as

$$
H=t^{-1}(0) t^{\prime}(0)=t^{\prime}(0) t^{-1}(0)
$$

where the prime denotes differentiation with respect to the variable $\lambda$. Furthermore it is possible to see that

$$
t^{-1}(0) t^{\prime}(0)=\sum_{i=1}^{N} h_{i(i+1)}
$$

where

$$
h=\left.\frac{d}{d \lambda}(P R(\lambda))\right|_{\lambda=0},
$$

and $N+1 \equiv 1$.

Thus we have defined a Hamiltonian that is the sum of local two-site Hamiltonians acting on a onedimensional lattice with periodic boundary conditions. Most integrable models on one dimensional lattices, such as the XXZ or anisotropic Heisenberg model [4], the supersymmetric t-J model [15-19] and the U model[20, 21], fall into the above description. Within the framework of the QISM in order to obtain solutions of particular models and get the spectrum of the commuting family of operators $t(\lambda)$ one uses the algebraic Bethe ansatz(ABA) [6,9]. The ABA provides a rigorous method for obtaining the Bethe ansatz equations. As the main interest here is to reveal some of the interplay between quantum groups and quantum integrable systems we refer to the excellent literature available $[6,9]$ for the details on the ABA.

\section{Multiparametric Integrable Systems}

Here we show how to obtain multiparametric quantum spin chains using Reshetikhin's construction [22] for multiparametric quantum algebras. We also demonstrate that under appropriate constraints these models may be transformed to quantum spin chains with twisted boundary conditions [12].

Let $(\mathcal{A}, \Delta, R)$ denote a quasitriangular Hopf algebra where $\Delta$ and $R$ denote the co-product and $\mathrm{R}$ matrix respectively. Suppose that there exists an element $F \in A \otimes A$ such that

$$
\begin{aligned}
(\Delta \otimes I)(F) & =F_{13} F_{23}, \quad(I \otimes \Delta)(F)=F_{13} F_{12}, \\
F_{12} F_{13} F_{23} & =F_{23} F_{13} F_{12}, \quad F_{12} F_{21}=I
\end{aligned}
$$

Theorem 1 of [22] states that $\left(\mathcal{A}, \Delta^{F}, R^{F}\right)$ is also a quasitriangular Hopf algebra with co-product and Rmatrix respectively given by

$$
\Delta^{F}=F_{12} \Delta F_{21}, \quad R^{F}=F_{21} R F_{21} .
$$

In the case that $(\mathcal{A}, \Delta, R)$ is an affine quantum algebra we have from [22] that $F$ can be chosen to be

$$
F=\exp \sum_{i<j}\left(H_{i} \otimes H_{j}-H_{j} \otimes H_{i}\right) \phi_{i j}
$$


where $\left\{H_{i}\right\}$ is a basis for the Cartan subalgebra of the affine quantum algebra and the $\phi_{i j}, i<j$ are arbitrary complex parameters. For our purposes we will extend the Cartan subalgebra by an additional central extension (not the usual central charge) $H_{0}$ which will act as a scalar multiple of the identity operator in any representation.

Suppose that $\pi$ is a loop representation of the affine quantum algebra. We let $R(\lambda), R^{F}(\lambda)$ be the matrix representatives of $R$ and $R^{F}$ respectively, which both satisfy the Yang-Baxter equation

$$
R_{12}(\lambda-\mu) R_{13}(\lambda) R_{23}(\mu)=R_{23}(\mu) R_{13}(\lambda) R_{12}(\lambda-\mu) .
$$

If $\left.R(\lambda)\right|_{\lambda=0}=P$ with $P$ the permutation operator then $\left.R^{F}(\lambda)\right|_{\lambda=0}=P$ as a result of (6). We may construct the transfer matrix

$$
\begin{aligned}
& t^{F}(\lambda)=\operatorname{tr}_{0}\left(\pi^{\otimes(N+1)}\left(I \otimes \Delta_{N}^{F}\right) R_{01}^{F}\right) \\
= & \operatorname{tr}_{0}\left(R_{0 N}^{F}(\lambda) R_{0(N-1)}^{F}(\lambda) \ldots . R_{01}^{F}(\lambda)\right),
\end{aligned}
$$

where $\Delta_{N}^{F}$ is defined recursively through

$$
\begin{aligned}
\Delta_{N}^{F} & =\left(I \otimes I \ldots \otimes \Delta^{F}\right) \Delta_{N-1}^{F} \\
& =\left(\Delta^{F} \otimes I \ldots \otimes I\right) \Delta_{N-1}^{F} .
\end{aligned}
$$

The subscripts 0 and $1,2, \ldots, N$ denote the auxiliary and quantum spaces respectively and $\operatorname{tr}_{0}$ is the trace over the zeroth space. From the Yang-Baxter equation it follows that the multiparametric transfer matrices $t^{F}(\lambda)$ form a commuting family. The associated multiparametric spin chain Hamiltonian is given by

$$
\begin{aligned}
H^{F} & =\left.\left(t^{F}(\lambda)\right)^{-1} \frac{d}{d u} t^{F}(\lambda)\right|_{\lambda=0} \\
& =\sum_{i=1}^{N-1} h_{i, i+1}^{F}+h_{N 1}^{F},
\end{aligned}
$$

with

$$
h^{F}=\left.\frac{d}{d u} P R^{F}(\lambda)\right|_{\lambda=0} .
$$

Through use of (6) we may alternatively write

$$
\begin{aligned}
J_{N} & =G_{N-1} G_{N-2} \ldots G_{1}, \\
G_{i} & =F_{i N} F_{i(N-1)} \ldots F_{i(i+1)},
\end{aligned}
$$

we can define a new transfer matrix

$$
\begin{aligned}
& t(\lambda)=J_{N}^{-1} t^{F}(\lambda) J_{N} \\
& =\operatorname{tr}_{0}\left(\pi^{\otimes(N+1)}\left(I \otimes \Delta_{N}\right)\left(F_{10} R_{01} F_{10}\right)\right),
\end{aligned}
$$

where we have employed the convention to let $F$ denote both the algebraic object and its matrix representative. Through further use of (6) we may show that

$$
\begin{array}{r}
t(\lambda)=\operatorname{tr}_{0}\left(F_{10} F_{20} \ldots F_{N 0} R_{0 N}(\lambda) R_{0(N-1)}(\lambda)\right. \\
\left.\ldots R_{01}(\lambda) F_{10} \ldots . . F_{N 0}\right),
\end{array}
$$

and the associated Hamiltonian is given by

$$
\begin{aligned}
H & =\left.(t(\lambda))^{-1} \frac{d}{d u} t(\lambda)\right|_{\lambda=0} \\
& =\sum_{i=1}^{N-1} h_{i, i+1} \\
& +\left(F_{N(N-1)} \ldots F_{N 1}\right)^{2} h_{N 1}\left(F_{1 N} \ldots F_{(N-1) N}\right)^{2}
\end{aligned}
$$

where

$$
h=\left.\frac{d}{d u} P R(\lambda)\right|_{\lambda=0} .
$$

The above Hamiltonian describes a closed system where instead of the usual periodic boundary conditions we now have a more general type of boundary condition. The boundary term in the above Hamiltonian is a global operator; i.e. it acts non-trivially on all sites. However we can in fact interpret this term as a local operator which couples only the sites labeled 1 and $N$. It can be shown that the boundary term commutes with the local observables $h_{i, i+1}$ for $i \neq 1, N-1$. This situation is analogous to the closed quantum algebra invariant chains discussed in [23].

From the above construction we may also yield models with twisted boundary conditions by an appropriate choice of $F$. Recall that we extend the Cartan subalgebra by the central element $H_{0}$. Let this element act as $c I$ in the representation $\pi$ where $c$ is some complex number. If we now choose $\phi_{i j}=0$ for $i \neq 0$ in the expression (8) the matrix $F$ factorizes as $F=M_{1}^{-1} M_{2}$ with

$$
M=\exp \left(\sum_{i=1}^{l} c \phi_{0 i} H_{i}\right),
$$

and $l$ is the rank of the underlying quantum algebra $U_{q}(g)$. Using the fact that the R-matrix satisfies

$$
\left[R(\lambda), I \otimes H_{i}+H_{i} \otimes I\right]=0, \quad i=1,2, \ldots, l
$$

tells us that

$$
\left[R(\lambda), M_{1} M_{2}\right]=0 .
$$

In this case the Hamiltonian (15) reduces to

$$
H=\sum_{i=1}^{N-1} h_{i, i+1}+M_{1}^{2 N} h_{N 1} M_{1}^{-2 N}
$$

which is precisely the form for a system with twisted boundary conditions (see [24]).

\section{Final Remarks}

We are aware that in this short and highly biased review it is virtually impossible to convey all the richness involved in the study of quantum integrable systems and quantum groups. Nevertheless it is important to mention some of its highlights as the intersection with conformal field theories (see [25] and references therein). In fact from the scaling limit of integrable models it is possible to obtain information about conformal field theories. In particular, from finite-size corrections of 
the Bethe ansatz equations of some models it is possible to calculate such quantities as the central charge and the conformal dimensions.

Also due to the quest for an appropriate theory explaining high $T_{c}$ superconductivity there has been an increasing interest in integrable models describing correlated electrons. Examples of such models are the supersymmetric $t-J$ model [15-19], the $E K S$ [26] and the $U$ model[20, 21] that are generalizations of the Hubbard model(see [27, 28]), as well as models related to a Temperley-Lieb algebra [29-31]. More recently an integrable model of correlated electrons based on an so(5) algebra and showing off-diagonal long-range order has been proposed [32].

The future points towards a multitude of directions some of them being the investigation of reaction-diffusion models [33],the study of q-vertex operators[34], the relation of integrable models with quantum matrix models [35] and the recently proposed integrable ladder models [36].

\section{References}

[1] W. Heisenberg, Z. Phys. 38, 441 (1926).

[2] H. Bethe, Z. Phys. 71, 205 (1931).

[3] L. Onsager, Phys. Rev. 65, 117 (1944).

[4] C.N. Yang and C.P. Yang, Phys. Rev. 150, 321 (1966); 150, 327 (1966);151, 258 (1966).

[5] R.J. Baxter, Proc. Roy. Soc. A 289, 1359 (1978).

[6] L.D. Faddeev, E.K. Sklyanin, A. Takhtajan, Teor. Mat. Fiz. 40, 194 (1979).

[7] M. Jimbo, Lett. Math. Phys. 10, 63 (1985).

[8] V.G. Drinfeld, Proc. Int. Cong. Math., 798 (Berkeley) (1986).

[9] E.K. Sklyanin,in Nankai Lectures in Mathematical Physics, ed. Mo-Lin Ge, (Singapore: World Scientific), 63 (1992). V.E. Korepin, N.M. Bogoliubov and A.G. Izergin, Quantum Inverse Scattering Method and Correlation Functions (Cambridge: Cambridge University Press) (1993). V. Chari and A. Pressley, A Guide to Quantum Groups (Cambridge: Cambridge University Press) (1994). M.Chaichian and A. Demichev, Introduction to Quantum Groups (Singapore: World Scientific) (1996). C. Gomez, M. RuizAltaba and G. Sierra, Quantum Groups in TwoDimensional Physics (Cambridge: Cambridge University Press) (1996).

[10] M. R-Monteiro, I. Roditi, L.M.C.S. Rodrigues and S. Sciuto Phys. Lett. B 354, 389 (1995).

[11] A. Foerster, I. Roditi and L.M.C.S. Rodrigues, Mod. Phys. Lett. A 11, 987 (1996).
[12] A. Foerster,J. Links and I. Roditi, J. Phys. A 31, 687 (1998).

[13] J. Links, Lecture Notes on Statistical Physics, unpublished.

[14] G. W Delius, M. D. Gould and Y.-Z.Zhang, Nucl. Phys. B 432, 377 (1994).

[15] C.K. Lai, J. Math. Phys. 15, 1675 (1974).

[16] B. Sutherland, Phys. Rev. B 12, 3795 (1975).

[17] P. Schlottmann, Phys. Rev. B 36, 5177 (1987).

[18] F.H.L. Essler and V.E. Korepin, Phys. Rev. B 46, 9147 (1992).

[19] A. Foerster and M. Karowski, Phys. Rev. B 46, 9234 (1992); Nucl. Phys. B 396, 611 (1993).

[20] A.J. Bracken, M.D. Gould, J.R. Links and Y.-Z. Zhang, Phys. Rev. Lett. 74, 2769 (1995).

[21] K.E. Hibberd,M.D. Gould and J.R. Links, J. Phys. A 29, 8430 (1996).

[22] N. Reshetikhin, Lett. Math. Phys. 20, 331 (1990).

[23] J. Links and A. Foerster, J. Phys. A 30, 2483 (1997).

[24] H.J. de Vega, Nucl. Phys. B 240, 495 (1984); C.M. Yung and M.T. Batchelor, Nucl. Phys. 446, [FS] 461 (1995).

[25] V. Pasquier and H. Saleur, Nucl. Phys. B 316, 523 (1990).

[26] F.H.L. Essler, V.E. Korepin and K. Schoutens, Phys. Rev. Lett. 68 (1992) 2960; ibid. 70, 73 (1993).

[27] F.H.L. Essler and V.E. Korepin, Exactly Solvable Models of Strongly Correlated Electrons (Singapore: World Scientific) (1994).

[28] P.B. Ramos and M.J. Martins, J. Phys. A 30, L195 (1997).

[29] J. Links, J. Phys. A 29, L69 (1996).

[30] A. Foerster, J. Links and I. Roditi, Mod. Phys. Lett. A 12, 1035 (1997).

[31] A. Lima-Santos, I. Roditi and A. Foerster Int. J. Mod. Phys. A 13, 4309 (1998).

[32] A. Foerster, J. Links and I. Roditi, J. Phys. A 32, L441 (1999).

[33] F.C. Alcaraz, M. Droz, M. Henkel, V. Rittenberg, Annals of Phys. 230, 250 (1994).

[34] O. Foda, M. Jimbo, T. Miwa, K. Miki and A. Nakayashiki, J. Math. Phys. 35, 13 (1994).

[35] C.-W.H. Lee and S.G. Rajeev Phys. Lett. B 436, 91 (1998).

[36] Y. Wang, Phys. Rev. B 60, 9236 (1999). 